\title{
Evaporation as an ageing procedure prior to wood preservative biological testing: when standardization needs metrology
}

\author{
L. Martin^ and M.-F. Thévenon
}

Received: 21 May 2014 / Accepted: 7 July 2014

\begin{abstract}
The wood preservation laboratory of Cirad is accredited by COFRAC (French accreditation committee - accreditation No. 1-1686) for tests on (1) durability of wood and wood-based products; (2) protective efficacy of wood preservatives; (3) efficacy of termite control products. In order to test the efficacy of wood preservatives, non-durable wood blocks are treated using different product doses and exposed to the attack of xylophageous organisms (fungi, insects). To reproduce the ageing of treated wood blocks, some laboratory procedures are available. Amongst them, there is an evaporation procedure, reproducing the action of a warm air flow onto treated wood. This ageing step is very discriminant, as only the formulations fixing effectively the active ingredients will pass the biological test afterwards. This ageing by evaporation is described in the EN73 standard. Nevertheless, many points remain difficult to overcome. The tunnels used for the evaporation are all prototypes; as such equipment is not available currently on the market. So each laboratory has got its own tunnel device. Moreover the way to measure the temperature and speed of the air flow is very difficult to achieve considering the prescriptions of the EN73 standard. The EN73 standard is being revised by the European standardization group (CEN TC38) and despite the metrological aspects were crucial and inadequate in former version, they were not considered as they should. The wood preservation laboratory has forwarded remarks in order to supersede some points of the revision document in order to consider the metrological aspects. This is of main importance as the ageing procedure is commonly used prior to most of the biological test, and such tested products are put on the market based on the efficacy results.
\end{abstract}

Keywords: Accreditation; wood protection; evaporation; standardization; metrology

\section{Introduction}

Wood is a renewable material, with many possible enduses from craft and art to building purposes. Nevertheless, due to its biological origin, this natural material can be degraded by different kind of lignivorous organisms: bacteria, fungi (cubic, fibrous and soft rots), and insects such as some coleoptera larvae and termites. Some wood species are renowned for their natural durability, i.e. their natural intrinsic ability to resist to biological attack, such as teak ( Tectona grandis L.f) or ipe (Tabebuia spp.) amongst many other tropical timber species. When the natural durability of the wood is not sufficient enough to insure that the wood will remain safe and sound for its expected service life, it is then necessary to protect it from biological attack [1].

The wood preservation laboratory of Cirad (Agronomy Research International Centre for Development, France) is accredited by COFRAC (accreditation No. 1-1686) according to ISO 17025 [2] standard for tests on durability of wood and wood-based products, on protective efficacy of wood preservatives, and, on termite control products according to different European standards. When a

`Correspondence: luc.martin@cirad.fr wood treatment has to be tested in order to determine its efficacy before being put on the market, it needs to go through different chemical and physical tests, ageing and biological tests, as well as eco-toxicological evaluation. Depending on the use class where the wood will be utilized, different biological and ageing tests are required [3].

The EN73 [4] is an evaporative ageing procedure (also called wind tunnel test) prior to a biological efficacy assessment and it is required for any wood preservative product before being put on the market for sale [3]. This step is crucial to determine the biological threshold of the products towards the biological wood destroying agent, depending on the type of timber (softwood or hardwood), the application method (aspersion, dipping, autoclave treatment...) and the type of active ingredients used.

Former formulations, such as CCA (Copper Chromium Arsenic) had bonding and networking systems through different chemical mechanisms with the wood fibers $[5,6]$. For these formulations, leaching could still be a problem [7], but evaporation was not much challenging. However, since 2004, wood preservatives based on arsenic are not allowed anymore in Europe or in Northern America. The wood protection systems are since mainly based on 




Fig. 1. Wind tunnel of the wood preservation laboratory, Cirad.

organic active ingredients (or a mix of organic/inorganic active ingredients) which do not react directly with the wood fibers. The organic active ingredients are in a formulation, and it is essentially this formulation that allows the active ingredients to be fixed into the wood for a certain period of time. In this case, it is obvious that ageing procedures such as evaporation are of importance prior to biological effectiveness tests, such as fungal or termite tests.

The aim of this paper is, through the example of the EN73 evaporation standard example, to underline the link that should be done between establishing a standard and including the adequate metrology expectations in order to create a virtuous circle, rather than to be unable to reach the expected requirements of a standard.

\section{Equipment requirement for EN73}

In the EN73 standard, the operating equipment needed is a wind tunnel. The wind tunnel is described as: compartmented and fitted with devices for heating and distributing the air. The air shall be dust-free, shall not be polluted (to have no effect on the test results) and shall leave the tunnel without be able to re-enter. The heating and distribution devices shall be such that the temperature and air velocity are maintained constant and uniform in each compartment.

There is no dedicated standard "wind tunnel", and thus each laboratory performing the EN73 test has its own wind-tunnel prototype (Fig. 1).

On the other hand, it must also be clear that not many laboratories in Europe have those wind tunnels and in some cases, the prototypes were made by the same company and are exactly the same from one laboratory to another. So, in reality, the wind tunnel equipments are not very different from one another.

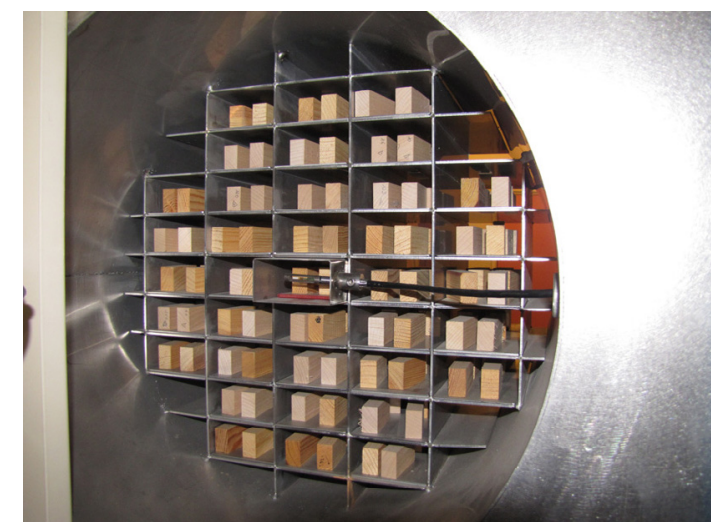

Fig. 2. Outlet of the compartments of the wind tunnel with the temperature and velocity regulation probe.

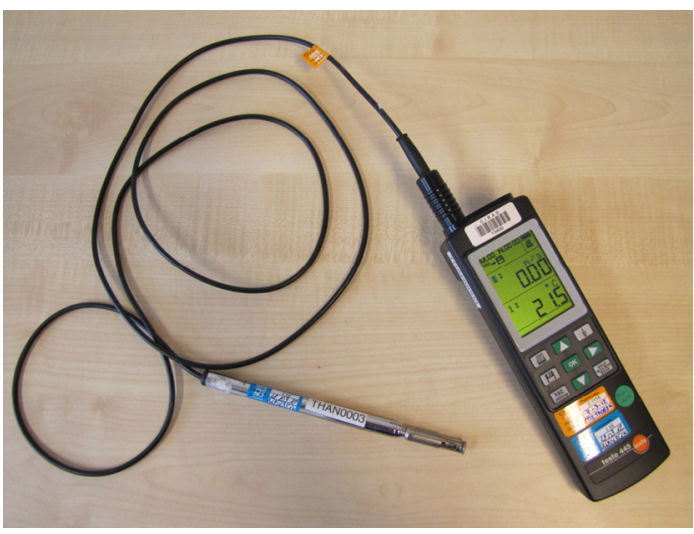

Fig. 3. Standard probe.

\section{Air flow requirement for EN73}

Once the treated wood samples are placed in the compartment of the wind tunnel, the air flow should be at a temperature of $(40 \pm 2){ }^{\circ} \mathrm{C}$, and at a velocity of $(1.0 \pm 0.1) \mathrm{m} / \mathrm{s}$. It is strictly specified that the air flow has to be controlled at this temperature and velocity at the outlet, on the axis of each compartment (Fig. 2).

\section{Air flow measurement requirements for EN73}

In order to measure the characteristics of the air flow, the requirements of the EN73 standard are: (1) a device which measures and records the air temperature; (2) an anemometer capable of measuring air velocity. The laboratory has got a standard probe able to measure and record both temperature and air velocity (Fig. 3). This standard probe is verified every 2 years by an external COFRAC accredited laboratory.

Measuring the temperature with the required tolerance is not a problem with this standard probe. Nevertheless, being able to measure the air flow at such a small tolerance is a real issue. First of all, finding this standard probe on the market was not an easy task. Moreover, this standard probe (for the anemometer) has a capability around 
2 only, which is a metrological limit to be confident of what is measured, as it is commonly admitted that a standard should have a minimum capability of 3 . In reality, such standard probe with a capability of 3 could have been found, but the price required for this option, including purchase and verification of the material, is extremely high. Thus, if the laboratory does respect the requirements of the EN73 standard from a strict metrological point of view, the cost of the standard probe traceability would be far too high to allow the wood preservation industry to pay for this ageing test. The laboratory would not offer competitive test prices anymore.

So, a question has to be pointed out: what is the benefit of this unrealistic requirement vs. the need for wood preservation industry in terms of both (1) determining the efficacy threshold of a wood protection product; (2) stay competitive on the market (when compared to non-wooden building products).

\section{Calibration of a wind tunnel}

In the wood preservation laboratory of Cirad, it has been decided to check and verify both of these parameters (temperature and air flow velocity at the outlet of each compartment) before any test, using untreated wood blocks as "lures". These lures wood samples are kept and used each time it is needed, different sizes and numbers being used according to the biological test to be performed afterwards [8-10]. This equipment qualification phase is exhaustive and time consuming. When a compartment is declared in line with the requirements (i.e. validation of the temperature and velocity parameters), a green label is put on the compartment and treated wood samples can go through evaporative test there. On the contrary, a red label is put on the compartment if this one is not in line with the EN73 requirements. In this case, only the lures will be maintained there during the 12 weeks of the ageing test.

Once the ageing test start, the treated samples in test are disposed in the tunnel, and some lures will stay if necessary. Over 12 weeks of test, the samples have to be rotated every week to have all the treated faces evaporated the same way (rotations will depend on how the test sample was treated: on one face only or on all faces) [8-10].

The Cirad wood preservation laboratory has got 4 wind tunnels, based on the exact same model, being build up at the same time by the same company and monitored the same way. Despite these facts, and over many years of tunnel calibrations, no rule or model could be found; leading then to an extensive time spent to make the calibrations prior to launching an evaporative test.

This calibration phase is also rather unrealistic from a metrological point of view for all the following reasons:

- the calibration is done at the outlet of the compartment where there is a turbulent flow regime;

- the calibration is done on lures and the test is done on treated samples of the same size, but even if marks are done on the grid of the compartments, the samples cannot be placed/replaced exactly at the same place;

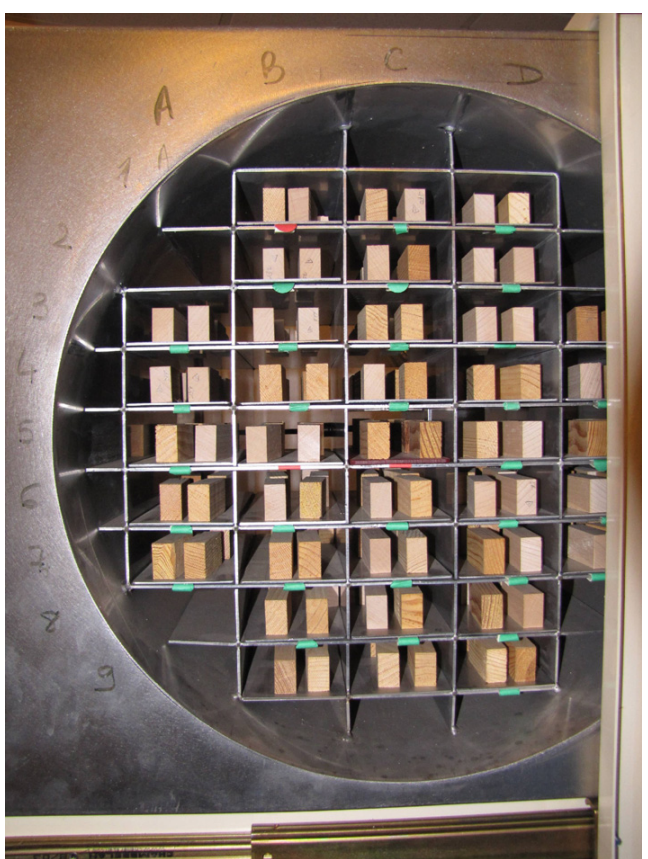

Fig. 4. Outlet of the compartments of the wind tunnel with green or red labels once the calibration is done.

- the same remark on the exact place of the sample is true each time the samples are rotated (i.e. once a week for 12 weeks).

\section{Discussion}

Considering all these facts, the team of the wood preservation laboratory, also participating to the European standardization committee, has forwarded all these remarks to the working group in charge of the EN73 standard revision. However, it is difficult to spot and change the challenging problems of this standard as (1) not many laboratories in Europe own wind tunnel equipments; and (2) most of these laboratories have an accreditation for this evaporative procedure (meaning that they can cope with this standard anyhow).

However, after several meetings, discussions and remarks, the revision project prEN73:2013 (document CEN/TC 38 N1957, 2013-12), the following major changes are:

- the anemometer has to be capable of measuring air velocity of $(1.0 \pm 0.3) \mathrm{m} / \mathrm{s}$;

- the temperature and air velocity should be measured and controlled when entering the compartments.

\section{Conclusion}

The example of the EN73 standard related here underlines that the metrology should be always taken into consideration when setting up or revising a standard. The ageing 
procedure is done prior to biological evaluation assessment from which is derived the biological threshold at which one the product should be used on the market.

Such metrological consideration in ageing or emissions procedures should be taken under high consideration. Wood preservative products are under scrutiny for their environmental impact on air $[11,12]$ and an harmonization in air flow measurements and associated tolerance should be more emphasized, metrology being one of the key factor for a realistic and objective evaluation of the impact of wood protection activity.

\section{References}

1. R.A. Zabel, J.J. Morrell, Wood Microbiology - Decay and its prevention (Academic Press INC, Editions Harcourt Brace Jovanovich, 1992)

2. EN ISO/IEC 17025, General requirements for the competence of testing and calibration laboratories (2005)

3. EN599-1, Durability of wood and wood-based products Efficacy of preventive wood preservatives as determined by biological tests, Part 1: Specification according to use class (2014)

4. EN73, Wood preservatives - Accelerated ageing of treated wood prior to biological testing - Evaporative ageing procedure (1988)
5. A. Pizzi, The chemistry and kinetic behavior of $\mathrm{Cu}-\mathrm{Cr}-$ As/B wood preservatives. I. Fixation of chromium on wood, J. Polym. Sci. Polym. Chem. Ed. 19, 3090-2131 $(1981)$

6. A. Pizzi, The chemistry and kinetic behavior of $\mathrm{Cu}-\mathrm{Cr}-$ As/B wood preservatives. II. Fixation of the $\mathrm{Cu} / \mathrm{Cr}$ system on wood, J. Polym. Sci. Polym. Chem. Ed. 20, 707-724 (1982)

7. EN84, Wood preservatives - Accelerated ageing of treated wood prior to biological testing - Leaching procedure (1997)

8. EN113, Wood preservatives - Test method for determining the protective effectiveness against wood destroying basidiomycetes - Determination of the toxic values (1996)

9. EN117, Wood preservatives - Determination of toxic values against Reticulitermes species (European termites, Laboratory method, 2013)

10. EN118, Wood preservatives - Determination of preventive action against Reticulitermes species (European termites, Laboratory method, 2014.)

11. XP ENV1250-1, Wood preservatives. Methods for measuring losses of active ingredients and other preservative ingredients from treated timber. Part 1: Laboratory method for obtaining samples for analysis to measure losses by evaporation to air (1995)

12. U. Schnoknecht, R. Wegner, W. Horn, O. Jann, Emission of biocides from treated materials, Env. Sci. Pollut. Res. 10, 154-161 